sense of security (4), difficulty moving the patient on a board (4), inappropriate use of the board (4), the non-conforming nature of the board (4), preventing proper examination (3), and scaring the patient (2).

\section{Discussion}

There is widespread use of spinal boards within the A\&E department. Advanced Trauma Life Support (ATLS) clearly states that the long board is for use "before and during transfer" and not for use within the A\&E department. This survey reveals that senior staff are well aware of the risks of spinal boards. However, boards remain the preferred method of immobilisation despite evidence that other surfaces are less hazardous and possibly give better support. ${ }^{2}$ The reasons for preference of the spinal board are not clear.

It is understandable that if a patient is brought to $A \& E$ on a spinal board then initial resuscitation should take priority over removal from the board. However, many clinicians delay removal well beyond this time. Even the best boards will affect the quality of $x$ rays taken through them-another reason for re- moval before the end of the primary survey. The patient will often have been on the board for at least 15-30 minutes before arrival in the $\mathrm{A} \& \mathrm{E}$ department and therefore tissue damage may already be occurring. Many place the patient on a board after arrival in A\&E. In these circumstances, the use of a vacuum mattress would be preferable. It may be that ATLS has introduced people to the spinal board but has not made them sufficiently aware of their problems or the alternatives available.

Hospitals should review their policies on use of spinal boards within the department using the evidence available to determine the best means of immobilisation within the $A \& E$ department.

1 Cooke MW. Spinal boards in A\&E. J Accid Emerg Med 1996;13:433.

2 Main PW, Lovell ME. A review of seven support surfaces with emphasis on their protection of the spinally injured. J Accid Emerg Med 1996;13:34-7.

3 Johnson DR, Hauswald M, Stockhoff C. Comparison of a vacuum splint device to a rigid backboard for spinal immobilisation. Am J Emerg Med 1996;14:369-72.

4 Carney C. Support surfaces. J Accid Emerg Med 1996;13: 302.

5 American College of Surgeons Advanced Trauma Life Support. Chicago: ACS, 1993.

\title{
Relatives in the resuscitation room: their point of view
}

F Barratt, D N Wallis

\section{Accident and}

Emergency

Department, Guy's and St Thomas' Trust,

London SE1

F Barratt

D N Wallis

Correspondence to: Dr F Barratt, Accident and Emergency Department,

University Hospital

Lewisham, Lewisham High

St, London SE13 6LH, UK.

Accepted for publication 22 October 1997

\begin{abstract}
Objective-To investigate whether bereaved next of kin would like to have been present in the resuscitation room during attempted cardiopulmonary resuscitation of their relative, and their experience or knowledge of what is involved in cardiopulmonary resuscitation.

Methods-The next of kin of patients who had recently died after unsuccessful cardiopulmonary resuscitation in the accident and emergency department were contacted initially by telephone and then sent a postal questionnaire.

Results-Four (11\%) of 35 respondents had been asked whether they wished to be present in the resuscitation room; 24 $(69 \%)$ would like to have been offered the opportunity, even though not all would have accepted. The respondents had a wide variety of perceptions of what happens during resuscitation, few of which corresponded to clinical practice.

Conclusions-Most relatives of patients requiring cardiopulmonary resuscitation would like to be offered the possibility of being in the resuscitation room; this could have several benefits.

$(\Im$ Accid Emerg Med 1998;15:109-111)
\end{abstract}

Keywords: relatives; cardiopulmonary resuscitation; resuscitation room
There has been recent debate ${ }^{1-3}$ over the presence of relatives in the resuscitation room, not only the parents of children but also relatives of adult patients. Most research has concentrated on the attitudes and feelings of medical and nursing staff, ${ }^{45}$ and in one study ${ }^{4}$ $75 \%$ of medical and nursing staff agreed with the statement, "Relatives should have the opportunity to be with a family member who is requiring cardiopulmonary resuscitation, provided appropriate professional support is available." By contrast little has been published on the attitude of recently bereaved relatives, although the Resuscitation Council (UK) published a report in 1996 with recommendations for practice and training. ${ }^{6}$

This study had two aims: first, to determine whether bereaved next of kin felt they would like to have been present in the resuscitation room during the attempted cardiopulmonary resuscitation of their relative; and second, to evaluate their knowledge and experience of what is involved in cardiopulmonary resuscitation.

\section{Methods}

The next of kin - as stated on the accident and emergency card - of patients over the age of 16 years who had died after unsuccessful cardiopulmonary resuscitation during a nine month period in the accident and emergency (A\&E) department of an inner city teaching hospital was contacted after a minimum interval of 
Table 1 Questionnaire to next of kin of patients who died after unsuccessful cardiopulmonary resuscitation

\begin{tabular}{|c|c|}
\hline Your name: & Your age: \\
\hline \multicolumn{2}{|c|}{ Name of Deceased: } \\
\hline \multicolumn{2}{|c|}{ Relationship: } \\
\hline \multicolumn{2}{|c|}{ Date of death: } \\
\hline Question 1 & $\begin{array}{l}\text { Did you witness what happened to your relative before they came } \\
\text { to hospital? }\end{array}$ \\
\hline Question 2 & Did you travel in the ambulance with your relative? \\
\hline Question 3 & $\begin{array}{l}\text { Were you asked whether you would like to be present in the } \\
\text { emergency room? }\end{array}$ \\
\hline Question 4 & Would you like to have been asked? \\
\hline Question 5 & Would you like to have been present? \\
\hline Question 6 & $\begin{array}{l}\text { Have you ever seen someone being resuscitated? (Not including } \\
\text { on TV) }\end{array}$ \\
\hline Question 7 & $\begin{array}{l}\text { What do you imagine happens in the emergency room when we } \\
\text { are trying to save someone's life? }\end{array}$ \\
\hline
\end{tabular}

three months. The survey was carried out in three stages. First, the next of kin was contacted by telephone; the study was explained and consent sought to send a questionnaire. Second, a simple questionnaire (table 1), together with a letter explaining the purpose of the study, was sent by post to those who had agreed to participate. Finally, one further letter was sent, either thanking respondents or requesting that non-respondents complete the questionnaire. Next of kin with no telephone number on the accident and emergency card were excluded, as were the relatives of patients who survived cardiopulmonary resuscitation.

Age and sex of respondents who expressed an unequivocal view as to whether or not they would like to have been present in the resuscitation room were analysed using a $t$ test and a $\chi^{2}$ test respectively.

\section{Results}

The next of kin of 78 patients could be contacted by telephone; $68(87 \%)$ agreed to a questionnaire being sent to them, and 35 $(51 \%)$ of 68 returned the questionnaire. The mean age of respondents was 58 years; 24 $(69 \%)$ were female and $11(31 \%)$ were male. Fifteen were spouse of the deceased, eight a son or daughter, two a parent, three a sibling, four a more distant relation, and three were indeterminate.

Table 2 shows the responses to questions 1 to 6 of the questionnaire. Seventeen (49\%) of 35 had witnessed their relatives collapse before transfer to hospital and nine (26\%) had travelled with their relatives in the ambulance. Four (11\%) had been asked on arrival in A\&E whether they wished to be present in the resuscitation room during the resuscitation of their relative; $26(74 \%)$ were not asked, and five $(14 \%)$ arrived after death. Twenty four $(69 \%)$

Table 2 Results of answers to questions 1 to 6 of questionnaire

\begin{tabular}{lrrll}
\hline & Yes & No & $\begin{array}{l}\text { Not } \\
\text { applicable }\end{array}$ & $\begin{array}{l}\text { Equivocal } \\
\text { answer }\end{array}$ \\
\hline Question1 & 17 & 18 & 0 & 0 \\
Question2 & 9 & 26 & 0 & 0 \\
Question3 & 4 & 26 & 5 & 0 \\
Question4 & 24 & 9 & 0 & 2 \\
Question5 & 15 & 14 & 0 & 6 \\
Question6 & 5 & 30 & 0 & 0
\end{tabular}

said they would like to have been asked if they wished to go into the resuscitation room, and of these, $15(62 \%)$ stated unequivocally that they would have chosen to be present. Five (14\%) had previously seen someone being resuscitated; one of these was a nurse.

Analysis of 29 respondents who expressed an unequivocal view as to whether or not they would like to have been present in the resuscitation room showed the mean age of those who would like to have been present was 56 years, compared with 65 years among those who would not $(p=0.1450)$. Nine $(60 \%)$ of the 15 who would like to have been present were female, compared with $11(79 \%)$ of the 14 who would not $(p=0.280)$. Six of nine men $(67 \%)$ nine of 20 women (45\%) would have liked to have been present, a difference of $22 \%$ (95\% confidence interval $-16 \%$ to $+60 \%$ ).

A wide variety of answers was given to the question "what do you imagine happens in the emergency room when we are trying to save someone's life?" Nine (26\%) mentioned "electric shock treatment," seven (20\%) "massage," seven (20\%) "mouth to mouth breathing," nine (26\%) "injection" and/or "adrenaline," and three (9\%) "oxygen." Five (14\%) used the phrase "various resuscitation methods"; one (3\%), a nurse, stated that she was familiar with most resuscitation procedures; nine (26\%) said "everyone did their best"; three (9\%) had "no idea"; and one (3\%) wrote "nothing was done." Two (6\%) gave answers that appeared to make little sense.

\section{Discussion}

In this study only four of 35 next of kin (11\%) were asked if they wished to go into the resuscitation room during the resuscitation of their relative, whereas the majority of respondents to the questionnaire said they would like to have been offered this opportunity (even if not all would have taken up the offer). There were no significant differences in age, sex, or relationship characteristics between those who would have liked to have been present and those who would not, but the numbers in this study were small.

Respondents did not have an accurate picture of cardiopulmonary resuscitation, and misconceptions among the public may have significant implications if relatives are to be present in the resuscitation room.

Possible sources of bias in this study were exclusion of the next of kin for whom a telephone number was not recorded and the next of kin of those who survived. The response rate to the questionnaire was relatively low ( $51 \%$ of those who agreed on the telephone), although this figure is in line with some reported response rates to postal questionnaires.

There are few documented examples of relatives being offered the opportunity to be present in the resuscitation room during cardiopulmonary resuscitation. At Foote Hospital - in Jackson, Michigan, USA - where there has been a programme of "planned participation of family members in resuscitation" since 1982, relatives are invited into the resuscitation room at a specified time in the 
resuscitation and are accompanied at all times. ${ }^{8}$ This practice has been accepted by all the staff involved in resuscitation. Relatives require full practical and emotional support, and occasionally have to be escorted out of the resuscitation room.

Several possible benefits might follow from relatives being present during cardiopulmonary resuscitation. They would see that everything possible was done ${ }^{68}$; fantasy may often be worse than reality. They might help the medical team to decide when to discontinue resuscitation. Being present during resuscitation could help the next of kin in coming to terms with the death of a relative, ${ }^{8}$ just as it is now accepted good practice to offer bereaved relatives the opportunity to see the dead body.

While doctors may have reservations about the presence of relatives during resuscitation, ${ }^{2}$ the Foote Hospital experience suggests that when appropriate support and supervision is provided some of these fears (such as interference by the relatives in resuscitation) may be exaggerated. ${ }^{8}$
In conclusion, we believe that a prospective study with larger numbers may help clarify the significance of our results. In the meantime the recent publication from the Resuscitation Council (UK) ${ }^{6}$ provides valuable guidelines for practice.

We would like to acknowledge assistance and advice from Dr E Glucksman.

1 Adams S, Whitlock M, Basket P, Bloomfield JF, Higgs R. Should relatives be allowed to watch resuscitation? BMJ 1994;308:1687-9.

2 Schilling RJ. No room for spectators [letter]. BMJ 1994;309:406.

3 Day J. Opportunity is appreciated [letter]. BMJ 1994;309: 670.

4 Back D, Rooke V. The presence of relatives in the resuscitation room. Nursing Times 1994;90(30):34-5.

5 Redley B, Hood K. Staff attitudes towards family presence during resuscitation. Accid Emerg Nursing 1996;4:14551

6 Should relatives witness resuscitation? A report from a project team of the Resuscitation Council (UK). London: Resuscitation Council (UK), 1996.

7 Barnet V. Sample survey: principles and methods, 2nd ed. London: Hodder and Stoughton, 1991.

8 Hanson C, Strawser D. Family presence during cardiopulmonary resuscitation: Foote Hospital emergency department's nine-year perspective. J Emerg Nursing 1992;18: ment's

\title{
Tick bite anaphylaxis in Australia
}

\author{
Anthony F T Brown, Duncan L Hamilton
}

Department of

Emergency Medicine, Royal Brisbane Hospital, Queensland 4029, Australia A F T Brown D L Hamilton

Correspondence to: Dr Anthony F T Brown, Staff Specialist, Department of Emergency Medicine, Royal Brisbane Hospital, Brisbane, Queensland 4029, Australia.

Accepted for publication 8 September 1997

\begin{abstract}
Tick bite anaphylaxis has rarely been reported. It may follow the bite of any of the different tick life cycle forms, is related to the release of salivary juices, and may range from mild itch to severe wheeze or shock. Data obtained suggest that it is more common and potentially life threatening than tick paralysis, which is more widely reported. Emergency physicians should recognise this possibility following a tick bite and be prepared to give treatment such as adrenaline rapidly. Patients should be referred to an allergist after recovery.

(F Accid Emerg Med 1998;15:111-113)
\end{abstract}

Keywords: anaphylaxis; tick infestations; tick borne diseases

Ticks and mites of the order Acari are specialised arachnids, successfully exploiting a wide variety of habitats. While mites are responsible for causing scabies, dermatitis including "grocers itch" or "grass itch," and various plant and animal infestations, ${ }^{1}$ ticks appear better known, particularly for causing paralysis. Eight hundred species of ticks are described internationally, with 70 species in Australia, ${ }^{2}$ of which at least 15 are known to have attacked man. Tick paralysis in Australia is caused by the common bush or scrub tick Ixodes holocyclus found in eastern coastal regions from northern Queens- land to Victoria, although occasional cases have been reported due to Ixodes cornuatus found in southern New South Wales, Victoria, and Tasmania. ${ }^{3}$ Adult female ticks are usually responsible, with the earliest symptoms following several days of engorging, ${ }^{4}$ most commonly in spring and summer during warm, moist weather. ${ }^{5}$ Up until 1945, 20 deaths from tick paralysis had been reported, mostly in children under three years of age. ${ }^{6}$

Tick paralysis is encountered worldwide, from North America, Western Canada, and Africa to Europe including the United Kingdom. ${ }^{7}$ Several different genera of tick are responsible including Dermacentor, Amblyomma, Ixodes, Haemaphysalis, Hyaloma, and Rhinicephalus, most often affecting animals such as dogs, sheep, and cattle. Fewer species are definitely implicated in human disease, which typically affects young girls during the spring and summer months or adult males exposed occupationally during farming or forestry work. $^{89}$ In addition to paralysis, ticks have many other important medical effects including bite site infection, foreign body granuloma due to retained mouth parts, local allergy including dermatitis, systemic anaphylaxis, and such vector borne diseases as Lyme disease, tularaemia, tick typhus, Rocky Mountain spotted fever, erhlichiosis, and babesiosis $^{59}$ (table 1$)$.

The ill effects of native Australian ticks were first recorded in 1827, with definite human 